Etnográfica

Revista do Centro em Rede de Investigação em

Antropologia

vol. $15(2) \mid 2011$

Vol. 15 (2)

\title{
Quand le mari de ma mère n'est pas mon père: beaux-enfants et beaux-pères dans le Brésil contemporain
}

Quando o marido da minha mãe não é meu pai : enteados e padrastos no Brasil contemporâneo

\section{Christine Jacquet}

\section{(2) OpenEdition}

\section{Journals}

Édition électronique

URL : https://journals.openedition.org/etnografica/943

DOI : 10.4000/etnografica.943

ISSN : 2182-2891

\section{Éditeur}

Centro em Rede de Investigação em Antropologia

Édition imprimée

Date de publication : 1 juin 2011

Pagination : 287-310

ISSN : 0873-6561

Référence électronique

Christine Jacquet, «Quand le mari de ma mère n'est pas mon père: beaux-enfants et beaux-pères dans le Brésil contemporain », Etnográfica [En ligne], vol. 15 (2) | 2011, mis en ligne le 26 octobre 2011, consulté le 10 février 2022. URL : http://journals.openedition.org/etnografica/943 ; DOI : https:// doi.org/10.4000/etnografica.943

\section{(c) (†) 8}

Etnográfica is licensed under a Creative Commons Attribution-NonCommercial 4.0 International License. 


\title{
Quand le mari de ma mère n'est pas mon père : beaux-enfants et beaux- pères dans le Brésil contemporain
}

\begin{abstract}
Christine Jacquet
S'inspirant de l'approche développée par Florence Weber sur la parenté pratique, cet article tente de cerner la nature du lien entre beaux-pères et beaux-enfants au Brésil, en présentant le système de justification adopté par ces derniers pour rendre compte des sentiments qu'ils nourrissent à l'égard de leur beaupère. La singularité de la parenté brésilienne - qui ne fait pas reposer la définition du parent uniquement sur la consanguinité et admet l'exercice effectif de la pluriparenté - nous incitait à penser a priori que le partage du quotidien était susceptible de faire naître chez les beaux-enfants des sentiments familiaux; les résultats de notre recherche ont toutefois largement démenti cette hypothèse initiale.
\end{abstract}

MOTS-CLÉS : recomposition familiale, parenté pratique, sentiments de parenté, affiliation volontaire.

\footnotetext{
"Lorsqu'il reprit connaissance, il se trouvait dans un lunch-room où on l'avait transporté en toute hâte pour le ranimer. Dans une glace ronde encadrée d'un cercle serré de petites ampoules électriques, il aperçut sa barbe blanche et son nez osseux. Au premier moment, il crut que cette barbe et ce nez appartenaient à quelqu'un d'autre. Il fallut qu'il vît le visage des siens qui l'entouraient pour se reconnaitre lui-même" (Joseph Roth, Le poids de la grâce).
}

SI DEPUIS LES ANNÉES 50, LES FAMILLES RECOMPOSÉES, QUI INTERROgent nos catégories de construction de la parenté, ont fait l'objet dans différents pays - parmi lesquels les États-Unis font figure de pionniers - d'une abondante littérature sociologique et anthropologique, au Brésil, par contre, elles n'ont pas connu le même succès; seuls les psychologues leur ont accordé un intérêt, mais dans une perspective d'évaluation où se côtoient les injonctions à l'équilibre, à la satisfaction et au bien-être. Or l'existence au Brésil de diverses formes d'affiliation volontaire semblerait propice - peut-on penser $a$ priori à titre d'hypothèse - à l'instauration d'un lien familial entre beau-père et 
bel-enfant. Nous souhaiterions tout d'abord revenir brièvement sur certaines caractéristiques de la parenté brésilienne, puisqu'elles ont guidé les premiers pas d'une investigation menée dans les états de Bahia et Sergipe qui prétendait observer la teneur du lien tissé entre beau-père et bel-enfant. Nous nous proposons ensuite d'exposer certains résultats de cette recherche - ceux qui nous ont le plus surpris.

\section{NORMES ET PRATIQUES FAMILIALES AU BRÉSIL}

Au Brésil, la catégorie de parent, qui d'une part n'est pas toujours circonscrite aux seuls géniteurs, d'autre part peut s'appliquer à plusieurs adultes simultanément, présente une certaine malléabilité, comparativement aux systèmes européens et américains de parenté tels qu'ils sont décrits par la littérature, et dont les deux piliers sont l'idéologie du sang et le principe d'exclusivité de la filiation (voir entre autres: Fine 1998, 2001 ; Le Gall et Bettahar 2001).

La circulation d'enfants entre foyers, qu'il s'agisse de transferts temporaires ou permanents, est loin d'être un fait récent ${ }^{1}$ ou marginal au Brésil. De nos jours aucune donnée quantitative n'est disponible qui permette d'évaluer avec quelque précision l'ampleur ou les caractéristiques du phénomène. ${ }^{2}$ Néanmoins, à partir de l'exploration de diverses enquêtes statistiques, ${ }^{3}$ Serra (2003) a tenté quelques estimations. La circulation est ici appréhendée par le nombre d'enfants âgés de 0 à 14 ans qui, au moment de la passation du questionnaire, ne résidaient pas avec leur génitrice, bien que cette dernière soit vivante; ont été exclus les enfants placés en institution ou vivant dans la rue. Selon les sources analysées, entre 6,5 et $9,1 \%$ des enfants appartenant à la tranche d'âge 0-14 ans sont confiés à autrui. Ces chiffres tendent toutefois à surestimer les transferts puisqu'ils incluent des enfants de couples séparés qui résident avec leur géniteur; or, selon une enquête de l'IBGE effectuée en 1985 uniquement dans les régions métropolitaines et auprès des mères, près d'un quart des enfants déplacés vivaient avec leur père. Cependant, comme le souligne Serra elle-même, toutes les données disponibles sont de nature transversale; elles écartent donc des comptages les enfants qui dans le passé ont

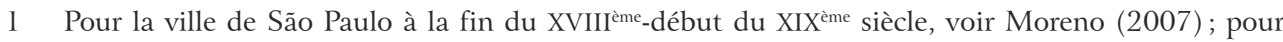
Porto Alegre au début du XX ${ }^{\text {ème }}$ siècle, voir Fonseca (2006b [1995]).

2 Lallemand (1993) remarquait déjà la difficulté à réunir des données quantitatives sur la circulation des enfants, pratique informelle et parfois illégale. Les données "portent souvent sur des milieux différents, généralement fort restreints" (Lallemand 1993: 42); de plus, les méthodes de recueil des informations ainsi que celles de calcul sont très hétérogènes.

3 Les PNAD (Pesquisa Nacional por Amostragem de Domicílios) de 1985, 1996, 1997, 1998 et 1999 réalisées par l’IBGE (Instituto Brasileiro de Geografia e Estatísticas); les Pesquisas Nacionais sobre Demografia e Saúde de 1986, 1991 et 1996 réalisées par la Benfam (Sociedade Civil do Bem-Estar Familiar no Brasil). 
connu une expérience de placement provisoire, avant de retourner vivre avec leur mère. De plus, ces données peuvent aussi sous-estimer certaines modalités de transferts, telles que "l'adoption à la brésilienne", dont nous reparlerons plus loin, et qui équivaut à une reconnaissance juridique frauduleuse de paternité/maternité.

Les travaux anthropologiques de Fonseca, tout en enregistrant la force de la "mystique autour des liens de sang" (2006b [1995]: 36), attestent que la circulation d'enfants entre adultes constitue "une structure de base de l'organisation de la parenté" (2006b [1995]: 15) dans les classes populaires urbaines où la prise en charge de la progéniture est une responsabilité collective: le rôle des géniteurs est de garantir à leur enfant des soins convenables, mais ceux-ci peuvent être assurés par autrui. Le placement des enfants chez un tiers, qu'il soit suscité par une situation de crise ou non, est pratiqué de préférence entre consanguins, mais s'étend aussi aux affins, voisins ou amis: "pour de nombreuses personnes, s'occuper d'un enfant est un sujet qui ne se limite pas à la mère, ni au couple. Cela mobilise un réseau d'adultes qui s'étend au-delà du groupe de parenté lui-même" (Fonseca 2002). Il ne conduit pas à l'éviction et à la substitution des géniteurs - l'identité familiale originelle de l'enfant est préservée - mais à un cumul de parents : de lait, d'élevage, véritables, etc., qui assument différentes fonctions parentales. Bien que la délégation de la prise en charge des enfants ne soit pas toujours exempte de rivalités entre les différentes mères, biologique et sociales, ${ }^{4}$ elle crée entre les partenaires du transfert - enfant, géniteurs et récipiendaires - des obligations morales sur la base desquels se tissent des liens durables. C'est pourquoi Fonseca (2002) propose, à titre d'hypothèse, que "comme dans beaucoup d'autres sociétés, ici la parenté ne se limite pas à l'aspect biologique, élément immanent à l'individu, mais est quelque chose qui se nourrit de la relation sociale tout au long de la vie".

Il est actuellement difficile de se prononcer sur l'extension sociale de la circulation des enfants au Brésil, faute d'informations consistantes. Les recherches de Fonseca ont porté exclusivement sur des groupes populaires de Porto Alegre ; l'auteur nous livre néanmoins une de ses intuitions - qu'aucune donnée ne vient toutefois étayer comme elle-même le souligne:

"malgré le manque de recherches systématiques dans ce domaine, je risque l'hypothèse qu'au cours de la dernière génération, les enfants de la classe moyenne ont cessé de circuler. [...] Avec sa mémoire opportunément courte, ce groupe a commencé à condamner la circulation des enfants comme une pratique de parents 'dénaturés', l'utilisant pour marquer la distinction entre

4 Les conflits émergent lorsque les mères n'appartiennent pas au même groupe de consanguins et lorsque l'enfant a été placé en bas âge. 
les familles respectables et les familles moralement répréhensibles" (Fonseca 2006b [1995]: 40).

Selon Abreu (2002), le transfert d'enfants se produit surtout dans les classes populaires et les fractions inférieures des classes moyennes. Les analyses statistiques de Serra (2003), précédemment mentionnées, nous incitent toutefois à ne pas radicaliser les différences entre l'organisation familiale des classes populaires et celle des classes moyennes - sans pour autant, bien sûr, conclure à leur similitude. En effet, si les mères dont un enfant est placé sont sans conteste surreprésentées dans les milieux populaires, leur proportion est toutefois loin d'être insignifiante dans les catégories moyennes ou supérieures. Il est, dans ces conditions, difficile de considérer le transfert d'enfants comme une pratique résiduelle, voire dépassée par les classes moyennes et supérieures, désormais ralliées au modèle nucléaire. En réalité, la mobilité enfantine parait davantage associée à la carrière conjugale et familiale de la mère (nombre d'unions, situation conjugale, âge lors de la naissance de l'enfant placé, nombre total d'enfants) qu'à sa seule position sociale.

Outre le fosterage, l'une des modalités de circulation enfantine signalée par la littérature anthropologique et juridique est, comme nous le mentionnions, l'adoption à la brésilienne, qui, selon Abreu (2002), représente la majorité de l'ensemble des adoptions réalisées au Brésil. Elle consiste à reconnaître juridiquement un enfant sans en être le/a géniteur/trice. Il s'agit en fait d'un don d'enfant réalisé le plus souvent en milieu d'interconnaissance; ainsi la mère biologique ne perd pas systématiquement tout contact avec son enfant, même si ce dernier méconnaît le lien qui l'unit à sa génitrice. Cette pratique, parce qu'elle simule la filiation biologique de l'enfant - à la manière de l'adoption plénière -, peut être lue comme un indicateur de l'importance de l'idéologie du sang partagé dans la définition brésilienne de la parenté. Néanmoins elle peut aussi être interprétée comme un moyen de contourner non seulement des procédures juridiques fastidieuses, mais aussi un cadre législatif resté longtemps très nettement discriminatoire à l'égard de la filiation adoptive, puisqu'elle permet à des adultes de faire rentrer un enfant de plein droit dans leur lignée familiale (Abreu 2002). ${ }^{5}$

5 Jusqu'à la promulgation en 1979 du Código de Menores, l'adoption relevait du droit privé: elle était formalisée par un contrat enregistré au cartório, entre les géniteurs, détenteurs de la puissance parentale, et un tiers. Elle s'opérait sans rupture des liens avec les parents biologiques; la parenté résultant de l'adoption n'avait des effets légaux qu'entre l'adoptant et l'adopté et le registre de naissance original de l'enfant était maintenu. Les enfants adoptés ne bénéficiaient pas des mêmes droits que les enfants légitimes, notamment en matière d'héritage. L'adoption comme transaction privée sera abrogée en 1990, date d'entrée en vigueur de l'ECA (Estatuto da Criança e do Adolescente) qui prévoit que les procédures passent désormais exclusivement par l'Etat. 
Illégale, l'adoption à la brésilienne est considérée comme un crime (art. 242 du code pénal), passible d'une peine de réclusion variant entre deux et six ans; cependant la loi se montre bienveillante si le crime résulte d'un acte "de noblesse reconnue" : est prévue alors une peine de détention de un à deux ans, que le juge peut d'ailleurs décider de ne pas appliquer en accordant un pardon judiciaire; toutefois, dans tous les cas, une action civile publique peut être entamée afin d'annuler l'acte de naissance de l'enfant et donc sa filiation. Néanmoins, cette règle est loin d'être appliquée par les tribunaux qui se montrent indulgents: comme le remarque Abreu (2002: 53), "la grande majorité des professionnels du droit ne perçoit pas les irrégularités concernant l'adoption, comme un acte criminel. Pour beaucoup d'entre eux, chaque adoption est l'occasion pour un couple de trouver 'l'enfant que la nature lui a refusé'". De plus, le contenu juridique du lien de filiation a été profondément altéré au cours des deux dernières décennies: l'affection comme fondement légal des relations familiales a fait son entrée sur la scène juridique avec la Constitution de $1988 .{ }^{6}$ Depuis lors, le principe de filiation socio-affective, ${ }^{7}$ même s'il n'est pas inscrit dans la législation brésilienne, a peu a peu gagné du terrain, ainsi que le montre l'évolution de la jurisprudence relative aux conflits de paternité (Anderle 2002). D'ailleurs une proposition de loi déposée en 2007 au parlement (projet n. ${ }^{\circ} 2285$, Estatuto das Famílias) intègre pleinement la socio-affectivité comme fondement de la parenté, au même titre que la consanguinité et l'affinité. ${ }^{8}$ Ainsi, actuellement, coexistent deux "vérités" que les juges peuvent invoquer pour définir la paternité juridique: la "vérité" biologique établie par les tests d'ADN et la "vérité" socio-affective attestée par la possession d'état. Cette double référence en matière d'établissement de la filiation légale introduit des contradictions notables dans les décisions prises par les juges; mais il importe surtout pour notre propos de souligner que l'origine biologique d'un enfant a cessé d'être l'élément exclusif à partir duquel est définie sa filiation juridique. Certains doctrinaires considèrent d'ailleurs que la connaissance des

6 La Constitution de 1988 reconnaît les unions de fait comme des entités familiales et, ce faisant, supprime la distinction entre enfants légitimes (nés dans le mariage) et illégitimes (nés hors mariage). Elle met sur un pied d'égalité filiation biologique et filiation adoptive et garantit à tous les enfants le droit à la filiation paternelle.

7 Pour être admise comme filiation juridique, la filiation socio-affective doit résulter d'un acte volontaire et être attestée par des preuves tangibles démontrant la possession d'état. Selon Anderle (2002), la notion de possession d'état est très secondaire dans le droit brésilien puisqu'elle n'apparait que dans un seul article du code civil (article 203) et se réfère exclusivement aux personnes décédées et ayant vécu en union maritale ("Art. 203: O casamento de pessoas que faleceram na posse de estado de casadas não pode se contestar em prejuízo da prole comum, salvo mediante certidão de registro civil, que prove que já era casada alguma delas, quando contraiu o matrimônio impugnado"); toutefois, elle est actuellement amplement débattue par la doctrine et mobilisée par les tribunaux appelés à statuer sur les conflits de paternité.

8 Art. 10: "o parentesco resulta da consanguinidade, da socio-afetividade e da afinidade". 
origines biologiques relève des droits de la personnalité et doit être sans effet sur la filiation lorsque celle-ci est déjà constituée. ${ }^{9}$ La nouvelle loi sur l'adoption, récemment promulguée (loi n. ${ }^{\circ} 12.010$ du 3 août 2009), va dans le même sens en reconnaissant aux enfants adoptés le droit d'accéder au dossier de leur adoption et à connaitre leurs origines biologiques dès l'âge de 18 ans (art. 48). Il convient cependant de mentionner que même avant cette nouvelle loi, le secret absolu sur les origines des enfants légalement adoptés n'a jamais été mis en pratique de façon rigoureuse au Brésil, à la différence des pays européens ou des États-Unis (Yngvesson 2007) : au cours de la procédure d'adoption, les parents adoptifs ont accès à l'identité des géniteurs et au certificat de naissance original de l'enfant.

La situation juridique du beau-parent est elle aussi empreinte d'une certaine confusion : le droit contemporain brésilien hésite entre reconnaissance et négation. Depuis le code civil de 2002, beaux-parents et beaux-enfants sont considérés comme juridiquement apparentés : ils sont liés par une relation d'affinité en ligne directe au premier degré qui perdure après la dissolution de l'union, que cette dernière soit matrimoniale ou consensuelle (code civil, art. 1.595). ${ }^{10}$ Une telle définition pourrait avoir des conséquences en matière d'obligation alimentaire puisque, selon l'article 1.694 de ce même code civil, "les parents, les conjoints ou compagnons peuvent se demander les uns aux autres les aliments dont ils ont besoin pour vivre d'une façon compatible avec leur condition sociale, y compris pour faire face aux besoins de leur éducation ". En vertu de cette norme, différents beaux-enfants ont d'ailleurs intenté une action en justice contre leur beau-parent; néanmoins les juges se montrent réticents à les suivre, considérant soit que le terme "parents" utilisé dans l'article 1.694 se réfère exclusivement aux consanguins (alors que ce même code assimile très explicitement l'affinité à la parenté), soit qu'il se rapporte aussi aux affins mais ces derniers ne peuvent être soumis à l'obligation alimentaire que de façon subsidiaire et complémentaire aux consanguins. En outre, la possibilité pour le beau-parent d'exercer une autorité parentale est explicitement écartée par l'article $1.638 \mathrm{du}$ code civil : il stipule que l'autorité parentale du parent qui contracte une nouvelle union doit être exercée "sans aucune interférence du nouveau conjoint ou compagnon"; en fait, la garde, la tutelle ou l'adoption plénière constituent les seuls instruments légaux dont dispose le beau-parent pour créer des liens juridiques avec l'enfant de son conjoint. ${ }^{11}$ Mais récemment, la loi 11.924 du 17 avril 2009 autorise le bel-enfant à demander au juge que

9 Voir par exemple Lôbo (2003).

10 Signalons que dans le code civil antérieur, de 1916, l'affinité ne créait pas de parenté.

11 Contrairement à la garde, la tutelle et l'adoption supposent la perte ou la suspension de l'autorité parentale. L'article 91 du nouveau projet de loi n. ${ }^{\circ} 2285$, Estatuto das Famílias, permet au beau-parent de partager cette autorité avec le ou les parents de l'enfant quelle que soit le statut juridique de l'union recomposée, ou l'orientation sexuelle des partenaires. 
son certificat de naissance soit altéré de façon à accoler à son nom de famille, celui de son beau-père ou de sa belle-mère, après accord de ces derniers; cette altération n'a toutefois aucune incidence en matière de filiation légale.

En somme, l'idéologie du sang partagé constitue sans conteste le point d'ancrage fondamental de la parenté au Brésil. Fondamental mais non exclusif: l'observation des pratiques familiales met en évidence des formes d'affiliations électives et de pluriparenté non instituées qui relèvent d'arrangements privés, mais qui n'en sont pas moins effectives. Le droit, quant à lui, toujours hostile à remettre en cause la norme d'exclusivité du lien de filiation, semble pourtant dorénavant davantage soucieux de s'adapter aux pratiques familiales, en faisant coïncider filiation et parentalité et en dissociant filiation et origine biologique. La définition juridique de la parenté initialement circonscrite à la consanguinité (code civil de 1916) s'est progressivement étendue à l'affinité (code civil de 2002) et tend de nos jours à inclure, de fait sinon de droit, la socio-affectivité.

Ces quelques éléments brièvement rappelés ont servi à bâtir les hypothèses de travail d'une recherche qui visait à comprendre le(s) sens que beau-père et bel-enfant donnent à leurs relations. ${ }^{12}$ En l'absence d'assises biologique et juridique, le partage du quotidien est-il susceptible de faire naître chez les beaux-parents et les beaux-enfants, des sentiments familiaux, plus spécifiquement paternel et filial? La singularité de la parenté brésilienne qui, comme nous l'avons montré précédemment, ne fait pas reposer la définition du parent uniquement sur la consanguinité et admet l'exercice effectif de la pluriparenté, nous incitait à répondre par l'affirmative. De façon complémentaire, nous nous proposions de suivre les indications de Segalen (2000), à savoir "réfléchir au poids respectif des liens familiaux créés par le sang en regard des liens sociaux créés par le contact quotidien", en prenant garde de ne pas opposer ces deux registres - biologique et social - mais plutôt de voir comment ils s'agencent. Pour répondre à ces différentes questions, nous avons mené, en 2007 et 2009, dans les états de Bahia (Salvador et Feira de Santana) et Sergipe (Aracaju), une série d'entretiens individuels semi-directifs auprès de mères gardiennes $(n=25)$, de beaux-pères $(n=11)$ et de beaux-enfants $(n=19)$, dont certains appartenaient à la même famille recomposée. Tous les interviewés vivaient au moment de l'enquête, ou avaient vécu par le passé, une expérience de recomposition familiale à la suite d'une séparation ou d'un divorce (et non d'un veuvage), au cours de laquelle le temps de cohabitation entre beau-père et bel-enfant était, ou avait été, d'au moins deux ans. Les milieux sociaux d'appartenance des

12 Cette étude, financée par le CNPq (Conselho Nacional de Desenvolvimento Científico e Tecnológico) et la Fapitec (Fundação de Apoio à Pesquisa e à Inovação Tecnológica do Estado de Sergipe), a été menée avec la collaboration de boursiers d'initiation scientifique: Alécio Gama dos Reis, Michelle Christie Andrade Melo, João Alex Alves de Moura, Marcele Mathias de Souza et Renata da Silva Sousa. 
enquêtés ont été diversifiés sur la base de la profession ou, à défaut, du niveau scolaire de la mère gardienne ou, le cas échéant, du beau-père. Léchantillon a été construit à partir des réseaux de connaissances des différents membres de l'équipe de recherche, mais aussi de ceux des interviewés eux-mêmes. Les entretiens qui privilégiaient des informations de nature biographique, ont eu une durée minimum de deux heures; la plupart de ceux réalisés auprès des mères et des belles-filles ont largement dépassé ce temps et se sont déroulés en deux sessions. L'âge des beaux-enfants interviewés s'échelonne entre 14 et 28 ans ; cet échantillon qui devait initialement compter un nombre égal de filles et de garçons, est en fait composé d'une majorité de filles $(n=12)$, celles-ci s'étant montrées davantage disposées à participer à l'enquête. ${ }^{13}$

\section{UNE PARENTÉ NIÉE}

Les résultats de notre recherche se sont avérés surprenants dans la mesure où ils ont partiellement réfuté nos prévisions. En effet, si mères et beaux-pères se sont montrés disposés à qualifier de paternel le lien beau-parental, en revanche les beaux-enfants n'ont exprimé que très exceptionnellement un sentiment de nature filiale, voire familiale, à l'égard de leur beau-père, y compris lorsque la communauté de vie était ancienne. Ce qui nous a déconcertés, c'est moins la dissonance entre les récits des uns et des autres ${ }^{14}$ que les jugements à la fois exclusifs et catégoriques des beaux-enfants, contraires à nos attentes. C'est pourquoi nous souhaiterions dans la suite de cet article, centrer notre attention sur les beaux-enfants et présenter le système de justification qu'ils ont adopté pour rendre compte des sentiments qu'ils nourrissent à l'égard de leur beaupère; cela nous conduira à porter notre attention sur les logiques d'échanges entre beaux-pères et beaux-enfants - du point de vue de ces derniers -, et à les rapprocher de celles en vigueur au sein de la parenté. Pour ce faire, nous nous appuierons sur les outils analytiques proposés par Florence Weber (2002, 2003, 2005, 2006a, 2006b) qui appréhende, à la suite des recherches de Bourdieu, la parenté comme un ensemble de pratiques; en différenciant les appartenances objectives des appartenances ressenties, cette approche permet de prendre au sérieux, au-delà des liens officiels tels que définis par le droit, les sentiments moraux de parenté.

Prenant acte de l'ambigüité de la notion de famille, Florence Weber préfère recourir à trois concepts issus de l'anthropologie pour penser les relations familiales contemporaines: la parentèle, la lignée et la maisonnée constituent

13 De même, pour l'échantillon des adultes: nous avons rencontré une bonne acceptation de la part des mères et une certaine résistance de la part des beaux-pères qui nous renvoyaient toujours à leur conjointe pour procéder à l'entretien.

14 Johnson (2000) et Cadolle (2000) signalent aussi des discordances entre les entretiens menés auprès des enfants et ceux menés auprès des parents et beaux-parents. 
autant de modèles d'échanges au sein de la parenté, basés sur des contraintes morales - institutionnalisées ou non - et des sentiments de parenté distincts. La parentèle, définie comme un réseau de relations égocentré, fonctionne sur le principe de la réciprocité et suppose des sentiments de nature élective. La lignée, quant à elle, correspond à un groupe "de longue durée, [qui] unit des vivants et des morts à travers la propriété collective de biens symboliques (typiquement un nom propre collectif, des ancêtres prestigieux, un patrimoine inaliénable)" (Weber 2005: 214); les échanges s'effectuent ici selon l'impératif de transmission de ce capital et les sentiments relèvent de l'affiliation symbolique. Dans les systèmes à filiation indifférenciée, tel que celui des sociétés occidentales, un individu appartient objectivement à deux lignées : celle de son père et celle de sa mère. Néanmoins, cette double appartenance ne va pas toujours de soi : certaines formes d'unifiliation ont été observées par exemple dans les lignées aristocratiques ou d'entrepreneurs capitalistes, ou encore dans des familles monoparentales; en outre, un individu peut être adopté par les lignées de son conjoint, comme dans les cas de mariages en gendre. Enfin la maisonnée désigne un groupe de parenté instable, mobilisé autour d'un objectif commun, et dont les membres partagent des ressources qui sont redistribuées en fonction des besoins de chacun; elle est sous-tendue par une logique de solidarité et repose sur des sentiments d'attachement quotidien. Soulignons que la notion de maisonnée ne recouvre pas nécessairement celle de groupe domestique puisque son unité ne repose pas sur la cohabitation, mais sur "la conscience d'un bien collectif, d'importance variable selon les membres du collectif et selon les périodes, qui peut consister en l'entretien d'une maison dont la jouissance est partagée ou en le bien-être d'une ou plusieurs personnes collectivement chéries" (Weber 2003: 24).

Suivant ce schéma analytique qui appréhende la parenté à la fois comme groupes d'appartenance et comme réseau de relations, nous nous proposons donc d'examiner si - et comment - les beaux-enfants incluent, ou au contraire écartent, leur beau-père de leur lignée, de leur maisonnée ou de leur parentèle ; cela nous permettra de saisir la représentation qu'ils ont des liens de parenté.

\section{Des échanges comptables}

Les beaux-enfants de notre échantillon conçoivent les relations conjugales entre leur mère et son nouveau conjoint comme un système d'obligations morales, indépendant de la formalisation de l'union, entre des partenaires liés par un sentiment électif mais aussi par leur appartenance commune à une même maisonnée. Ces obligations sont de deux ordres. Affectif tout d'abord. Elles sont alors résumées par le terme "companheirismo" qui renvoie à des attributs de nature psychologique ou relationnelle (Bozon 1990; Singly 1994 [1987]): "dialogue", "respect", "soins", "affection", etc. Ces devoirs sont réciproques et non sexués: ils s'imposent aux deux conjoints avec la même intensité. 
Or nos interlocuteurs constatent une nette dissymétrie dans les échanges affectifs entre leur mère et son partenaire. Jessica par exemple souligne tous les "sacrifices" auxquels sa mère Jeane, professeure, a consenti afin de suivre son second conjoint, ingénieur pétrolier, amené à changer plusieurs fois d'état pour des raisons professionnelles :

"Elle avait une maison, elle avait toute une structure, elle avait ses amis, sa famille, tout dans la même ville et qu'on le veuille ou non, elle a tout laissé pour l'accompagner; elle a vécu dans trois états différents, elle a trouvé un travail, elle a commencé à s'adapter et lui il devait partir et de nouveau elle a dû recommencer tout le processus. Je pense qu'il avait le devoir de la soutenir davantage que ce qu'il a fait, je ne parle pas d'un soutien matériel mais émotionnel, il aurait dû davantage la soutenir".

Les sacrifices, formes extrêmes de dons, sont demeurés au fil du temps sans contrepartie équivalente. Les sentiments électifs qui fondent les relations entre conjoints, c'est-à-dire entre membres d'une même parentèle, supposent une réciprocité interindividuelle directe à laquelle, selon les beaux-enfants, leur beau-père se soustrait volontiers.

Mais ce sont les obligations financières envers la maisonnée qui font l'objet, dans les récits, des développements les plus longs et des récriminations les plus virulentes de la part des beaux-enfants contre leur beau-père. Or, comme l'ont bien démontré Cadolle (2000, 2005) et Martial (2003, 2005), l'argent dit les configurations relationnelles à l'œuvre dans les familles recomposées: sa gestion constitue un excellent indicateur des places de chacun. Attardons-nous donc sur l'organisation financière des familles enquêtées.

Les récits des beaux-enfants convergent pour affirmer que le beau-père n'assume pas les dépenses liées à leur personne : habillement, santé, scolarité. Quelque soit la valeur de la contribution paternelle, les enfants sont à la charge de leur mère. ${ }^{15}$ La séparation du couple parental provoque systématiquement une mise au travail des femmes auparavant inactives, qui ne renoncent pas à leur emploi à la suite de leur nouvelle union; la présence d'enfant d'un premier lit légitime au contraire l'exercice d'une activité professionnelle face à leur conjoint. Les seules interruptions d'activité constatées dans notre échantillon de mères sont temporaires, elles se produisent lors de la naissance d'un enfant du nouveau couple et n'excèdent jamais les premiers mois de vie du nourrisson.

Les mères ne sont pas toujours seules pour endosser la charge financière de leur progéniture; elles peuvent bénéficier d'aides de différentes origines.

15 Sur ce point, tous les entretiens sont univoques, y compris ceux menés avec les adultes (mères et beaux-pères). Le beau-père n'assume pas les frais de ses beaux-enfants; en cas de difficultés financières de sa conjointe, il peut l'aider très ponctuellement à faire face à ces dépenses. 
Environ un tiers des pères participe à l'entretien de leurs enfants par le versement d'une pension alimentaire ou, plus rarement, par la prise en charge directe de certains frais. Les grands-parents paternels peuvent parfois, selon diverses modalités, prendre le relais financier de leur fils. Par exemple, le grandpère paternel d'Alessandra a versé à son ex-bru une pension pendant la première année qui a suivi la séparation conjugale; Bruno passe ses week-ends, du vendredi soir au lundi matin, et ses vacances chez ses grands-parents paternels qui financent tous les frais relatifs à sa scolarité. L'aide la plus substantielle est toutefois apportée par les ascendants maternels. Une de ses expressions caractéristique est l'hébergement temporaire à la suite de la dissolution de l'union, qui peut s'étendre sur plusieurs années, notamment pour l'enfant resté au domicile grand-parental après la remise en couple de sa mère. Ainsi Rodrigo a un an quand ses parents se séparent; il va alors habiter chez ses grands-parents maternels, en compagnie de sa mère et de sa sœur. Trois ans plus tard, sa mère déménage pour former une nouvelle union mais Rodrigo ne rejoindra le nouveau couple qu'au bout de deux ans. La cohabitation avec son beau-père lui devenant insupportable, il retourne vivre chez ses grands-parents à l'âge de $1 \mathrm{l}$ ans et y demeurera jusqu'à 18 ans. Notons que la participation du père et de la mère à l'entretien des enfants est rarement équivalente, de même que celle des familles maternelle et paternelle; la mère et sa lignée compensent souvent l'absence de soutien du côté paternel.

En outre, la solidarité familiale - tant en ligne maternelle que paternelle - a parfois, pour les bénéficiaires, un coût qui s'exprime en termes d'ingérence et de perte d'autonomie (Attias-Donfut, Lapierre et Segalen 2002), qu'il convient de ne pas sous-estimer sous peine de s'en tenir à une lecture idéalisée des liens familiaux. Lucia, hébergée par ses grands-parents maternels avec sa mère et son frère, a dû endurer pendant de nombreuses années les remarques désobligeantes sur son père et le traitement humiliant de la part de sa famille maternelle:

“- Quelle a été la réaction de la famille, quand ta mère s'est séparée?

- Tout le monde [famille maternelle] commentait, parlait, critiquait, principalement mon grand-père, parce qu'il n'a jamais voulu que mes parents se fréquentent, alors il lui jetait à la figure, 'tu vois ce qui se passe? Je t'avais dit que ça n'allait pas marcher et maintenant tu me reviens sans travail, avec des enfants en bas âge! Je vais t'aider parce que je ne vais pas te laisser au milieu de la rue', mais il lui imposait beaucoup de choses, ma mère a beaucoup souffert au début, elle a souffert jusqu'à ce qu'elle trouve un travail, je veux dire qu'elle a passé des mois à pleurer, à supporter des affronts, sans argent pour acheter du lait, c'est pépé qui l'achetait, et aussi à servir de domestique [...]. Pour nous [Lucia et son frère] aussi c'était difficile parce qu'on entendait beaucoup de commentaires négatifs sur mon père, parce que tout le monde pensait qu'il était irresponsable, qu'il était ceci, qu'il était 
cela et même s'il avait tort, on aimait pas entendre certaines choses, ça nous mettait très mal à l'aise parce qu'on savait que c'était notre père ".

La non-prise en charge par le beau-père des dépenses relatives à ses beauxenfants relève d'une norme de fonctionnement familial à laquelle tous nos interlocuteurs - sans exception - souscrivent: s'il a des devoirs envers sa partenaire, il n'en a aucun envers la progéniture de celle-ci. Cependant, bien que les beaux-enfants reconnaissent volontiers que leur beau-père n'a aucune obligation financière à leur égard, ils considèrent, par contre, qu'il en a vis-à-vis de la maisonnée qu'il a formée avec sa nouvelle conjointe et à laquelle ils appartiennent. Par son alliance, il est devenu l'homme la maison, remplaçant en ce sens - mais en ce sens seulement - leur père ; en tant que tel, il doit pourvoir, indépendamment de ses ressources matérielles et de ses charges, au logement et à l'alimentation, considérés comme frais communs de la maisonnée. Autrement dit, la règle d'équité retenue par les beaux-enfants pour répartir les charges entre les membres de la maisonnée est basée sur le statut au sein du groupe (Coenen-Huther, Kellerhals et Von Allmen 1994; Kellerhals et Languin 2008) ; les obligations matérielles des beaux-pères sont fondées sur un partage sexuellement différencié des activités entre les membres du couple.

Comme le signale Florence Weber, le fonctionnement d'une maisonnée repose sur le principe de solidarité - une solidarité de type organique puisque basée sur la division du travail -, qui inscrit chacun de ses membres, tenus au devoir de dévouement à l'égard du groupe, dans une "spirale des échanges" où toute comptabilité est exclue, étant donnée la symétrie des dettes morales: “impossible de dire qui doit le plus à l'autre, impossible de dire qui donne, qui reçoit et qui rend" (Weber 2005: 159). Or dans leur récit, les beaux-enfants donnent à voir des beaux-pères dont le comportement effectif n'est guère régulé par un esprit de solidarité, manifestant ainsi l'absence de tout sentiment d'attachement quotidien à leur beau-père. Suite à la seconde union de sa mère, Danilo va habiter dans une fazenda qui appartient à son beau-père Diego ; ce dernier assume totalement ses obligations statutaires telles que définies par les beaux-enfants: il prend à sa charge le logement et l'alimentation de la maisonnée.

“Quand on a déménagé, on est allé habiter à la campagne mais il [Diego] voulait que je m'occupe de son jardin, il avait des chevaux, des porcs, des poules, je ne sais pas quoi encore. Je m'en occupais tout seul, je m'en occupais mais je ne savais pas faire, il disait: 'ah! tu ne sais rien faire'. C'était une réalité totalement différente de celle que je connaissais parce que jusqu'alors ma réalité n'avait rien à voir avec une ferme. Même l'école, il ne voulait pas me laisser faire mes devoirs pour que je travaille sur sa ferme, et ça c'est perturber ma vie scolaire parce que je pouvais avoir des mauvaises notes mais je ne pouvais pas laisser le travail. Alors je le faisais mais les choses ont changé 
avec le temps, il a commencé à exiger davantage, il disparaissait, allait se promener et je restais seul à travailler, c'était comme si je devais travailler pour manger, il a dit 'je vais accumuler du pouvoir sur eux parce que je leur donne à manger', ce pouvoir qu'il pensait accumuler a fait que la relation est devenue critique".

Les liens entre Diego et Danilo sont vécus par chacun selon des références différentes. Le premier inscrit ses relations avec son beau-fils dans l'ordre de la réciprocité: l'entretien des enfants de sa conjointe ne constitue pas un devoir moral dérivé de sa position statutaire au sein de la maisonnée, mais un don personnel qui fonde une dette ; dans cette perspective, l'attente de contrepartie est légitime. Par contre, pour Danilo, les relations qu'il entretient avec son beaupère ne sauraient être régulées par le principe du donnant/donnant, puisque l'appartenance de Diego à la maisonnée qu'il a formée en s'unissant à sa mère interdit toute comptabilité des échanges entre les membres. Danilo, enjoint à sacrifier sa scolarité - injonction qui démontre que l'éducation des enfants de sa conjointe ne constitue pas, pour Diego, l'objectif commun de la maisonnée -, se sent enfermé dans un statut subalterne de quasi-domestique: il doit travailler contre de la nourriture; c'est pourquoi Danilo peut constater que son beaupère "a déstructuré la famille" : il a redéfini les places de chacun.

Une autre modalité mise en mots par les beaux-enfants de déni de l'appartenance des beaux-pères à leur maisonnée et de l'existence de tout lien nourricier, consiste à souligner l'insuffisante contribution de ces derniers à la survie collective, insuffisante en référence au statut qui leur est octroyé par l'alliance, au sein du groupe.

“- Il ne veut pas participer aux dépenses de la maison, il laisse tout à ma mère, elle paie le loyer, parfois elle paie l'eau, elle paie l'électricité et il ne veut avoir aucune dépense avec rien du tout.

- Il n'a pas de dépenses à la maison, c'est ça?

- Il n'est pas du genre à arriver à la maison et dire: 'je vais payer ceci, je prends la note et je vais la payer'. Il ne donne que si ma mère demande, tu comprends? Et encore quand ma mère demande il met des obstacles, il demande pourquoi".

Gabriela met directement en regard les contributions de son beau-père à la maisonnée et les bénéfices non-proportionnels qu'il tire de sa participation au groupe :

“ S'il a voulu rester avec ma mère, c'est plus par intérêt parce qu'avec ce qu'il gagne il ne pouvait pas entretenir la maison comme elle est entretenue. Je pense que c'est seulement par intérêt... Je ne vois pas quel avantage ma 
mère voit dans une relation comme celle-ci, parce que ses dépenses sont doublées, marché, supermarché, notes à payer, tu comprends? Parce que le seul argent sûr qui rentre c'est surtout celui de ma mère tu comprends? Parce que lui il est autonome et il a pris sa retraite avec seulement un salaire minimum et il travaille comme coiffeur, il a un petit salon là-bas dans le centre qu'il divise avec un ami, mais il y a des jours où il revient avec de l'argent et il y a des jours où il revient sans argent, ce n'est pas quelque chose de fixe. Je ne crois pas qu'il aime ma mère, je crois que pour lui ça a été tout bénéfice d'avoir ma mère".

Puisque le comportement des beaux-pères par rapport à la maisonnée est dicté par l'intérêt personnel et non par la solidarité, les échanges peuvent faire l'objet d'une comptabilité qui révèle le sens de la dette, à savoir la position de débiteur - et non de créancier - des beaux-pères vis-à-vis du groupe. Nos enquêtés se sentent alors pleinement autorisés à recouvrer la dette auprès de leur beau-père - ce qui ne manque pas de déclencher des conflits ouverts avec ce dernier, mais aussi parfois avec la mère elle-même; lorsque l'enquêteur demande à Rafael de qualifier ses relations avec son beau-père, il répond sans ambages: “c'est plus une relation de prestation de comptes", soulignant qu'il est en position de contrôler les échanges. En fait, la légitimité de l'autorité du beau-père sur la maisonnée dépend directement de sa capacité à assumer ses obligations statutaires à l'égard du collectif. En transgressant les règles de fonctionnement de la maisonnée, il cesse d'être reconnu par les beaux-enfants comme chef de la maison; son autorité sur le groupe n'est plus fondée, comme l'explique Mônica à propos de son premier beau-père avec lequel elle a vécu entre l'âge de 8 et 17 ans :

“A l'intérieur de la maison, il voulait être l'autorité. C'est ma mère qui payait tout, il ne payait que la nourriture et c'est ma mère qui payait tout le reste, on habitait dans un appartement loué et je n'étais pas d'accord que ma mère paye le loyer et voir une autre personne de l'extérieur qui voulait commander, tu comprends? On se disputait beaucoup à cause de ça ".

En outre, les actions de recouvrement de créances entreprises par les beauxenfants auprès de leur beau-père, sont à leurs yeux pleinement justifiées par leur propre dévouement au groupe, abondamment illustré dans leurs récits. Julia, 14 ans, relate dans le menu détail comment elle s'occupe de sa demisœur utérine âgée de 2 ans, pendant que sa mère est au travail et alors que son beau-père, père de l'enfant, s'en désintéresse totalement au point de ne pas acheter le lait nécessaire à la préparation de la blédine de sa fille. Nadia, âgée de 28 ans, et au chômage depuis quelques mois, décrit tous les services qu'elle rend aux différents membres de la maisonnée qui inclut, outre sa mère 
et son beau-père, trois descendants de ce dernier: un petit-fils trisomique, un fils adulte handicapé et une fille.

\section{Une alliance sans affiliation}

Comme nous l'indiquions précédemment, seul un cinquième de nos enquêtés considère leurs relations avec leur beau-père comme filiales. L'occurrence de cette catégorie est certes un peu plus fréquente (un tiers) quand la cohabitation s'est étendue sur de nombreuses années ( 10 ans ou plus) et a commencé alors que l'enfant était encore très jeune (moins de 6 ans), confirmant en cela les résultats de Cadolle (2000) et Martial (2003); mais elle reste, malgré tout, nettement minoritaire. Plutôt que de nous attarder sur les conditions qui facilitent, ou au contraire entravent, la reconnaissance de relations filiales par les beaux-enfants, voyons ce que recouvre, pour eux, ce qualificatif: se rapportet-il à une commune appartenance à une lignée?

"J'ai à la fois deux pères et deux beaux-pères" affirme Carla évoquant ainsi Carlos, son père biologique, Caio, son "beau-père qui est comme son père" et Célio, son "second beau-père". Carla a 23 ans au moment de l'entretien. Elle est la fille unique d'un couple qui se sépare quatre ans après sa naissance ; ses parents maintiendront d'excellentes relations. Les contacts entre Carla et son père sont très fréquents jusqu'à ce que, quelques mois après la rupture conjugale, il change de région d'habitation et s'installe à plusieurs milliers de kilomètres de distance ; les rencontres deviennent alors épisodiques mais régulières, elles se produisent pendant les vacances scolaires. A partir de l'âge de 6 ans, Carla vit avec sa mère et le second époux de cette dernière, Caio, qui n'a pas d'enfant. La vie commune avec ce premier beau-père durera jusqu'à la dissolution de l'union; Carla a alors 17 ans. A cette même époque, son père se remarie avec une jeune femme, mère d'un bébé. Deux ans plus tard, la mère de Carla se met en ménage avec Célio; l'union sera formalisée au bout de quelques mois. Carla décrit en détail le comportement paternel de Caio, son rôle nourricier, éducatif et affectif, ce qu'il lui a patiemment transmis au jour le jour et l'a façonné en tant que sujet (goûts, manières d'être, de penser, etc.): “je vois ce que je suis aujourd'hui et je vois beaucoup de son influence, ce qu'il a fait pour moi, la façon dont il m'a éduquée". Ce qui donne à la relation sa connotation paternelle, c'est surtout le soutien infaillible manifesté par Caio, son dévouement quotidien à la maisonnée qu'il a constituée autour de Carla, avec sa conjointe et la mère de cette dernière ("quelque soit l'heure, il était toujours là si besoin "). ${ }^{16}$ Carla décrit comme chaleureuses et suivies les relations avec les parents de Caio, qu'elle considère comme ses grands-parents. Le lien entre Carla et son premier beau-père, nourri par le dévouement de ce

16 La grand-mère maternelle de Carla n’a jamais habité avec la famille mais s'est toujours beaucoup occupée de sa petite-fille. 
dernier, s'est construit sur la base de l'élection mutuelle: “je l'ai adoré tout de suite la première fois, dès qu'on s'est vu on s'est très bien entendu, je crois que je l'ai aimé avant de savoir qu'il était le copain de ma mère. Jusqu'à maintenant elle plaisante en disant que la première fois qu'elle l'a amené à la maison il a passé plus de temps avec moi qu'avec elle". Néanmoins, parce qu'il repose sur l'élection et le partage du quotidien, le lien demeure instable: il dépend de la volonté des partenaires de l'échange, mais aussi des évènements de la trajectoire conjugale et familiale - présente et future - du beau-père et de la mère. Ainsi, Carla sent sa relation avec Caio menacée à deux reprises : tout d'abord lors de la séparation de sa mère et de son beau-père:

“Je savais qu'ils étaient sur le point de se séparer. Un jour il est venu me dire qu'il partait. J'ai été beaucoup plus affligée que pour la première séparation [celle de ses parents] parce que j'ai beaucoup vécu avec lui et il y avait aussi cette peur, parce qu'il n'était pas mon père mais mon beau-père, s'ils se séparaient, de ne plus avoir de relation entre moi et lui. Même si sur le moment il me disait que ça n'allait pas changer, et réellement ça n'a pas changé, je ne savais pas si réellement ça n'allait pas changer, on n'allait plus habiter dans la même maison. Alors j'ai été très triste pendant plusieurs jours... Je crois que j'ai été plus triste que quand ma mère s'est séparée de mon père, parce qu'avec ma mère et mon père j'étais très petite et je n'avais aucunne notion de ce qu'est une séparation, je n'associais pas, aujourd'hui mon père habite avec moi, demain non, je n'ai pas fait le lien dans ma tête. Et je crois aussi que je savais que mon père était mon père, le fait qu'il soit le mari de ma mère était seulement un détail, mais c'était mon père. Et mon beau-père était mon père, de la façon dont je le voyais à l'époque, seulement parce qu'il était le mari de ma mère".

Puis, quelques années plus tard, lors du nouvel engagement familial de Caio :

“- Il s'est remarié?

- Oui.

- Comment as-tu vécu ce moment?

- Ah! Au début il y a eu une petite pointe de peur. Une nouvelle famille, tout de suite après il a adopté un bébé. Quand j'étais là-bas [chez Caio] la dernière fois, j'y suis allée pendant les vacances, et c'était à l'époque où il était en train d'adopter, je l'ai connu [le bébé] et tout, ça donne une petite pointe... maintenant il a une nouvelle famille, avec un enfant, est-ce qu'il ne va pas... jusqu'alors j'étais la seule enfant... ça a été plus à cause de l'enfant... Mais rien n'a changé tu sais, c'est plus que ça donne une petite pointe d'insécurité". 
Carla n’a jamais ressenti une telle insécurité lorsque son père s'est remarié avec une femme qui avait un bébé; ni d'ailleurs quand, prenant le parti de sa nouvelle épouse, il refuse que sa fille vienne habiter chez lui, signalant ainsi que sa solidarité va, en priorité, à sa maisonnée conjugale : cette dernière situation a suscité des conflits qui ont duré plusieurs mois entre Carlos et Carla mais n’a jamais été perçue par cette dernière comme une menace pour la relation, que Carla juge "merveilleuse": "avec mon père il y a quelque chose de très fort parce qu'il est mon père ". Contrairement à son père vis-à-vis duquel Carla occupe une position généalogique inaltérable, son beau-père reste en dernière instance le mari de sa mère. L'instabilité potentielle de la relation au beau-père rend impossible tout sentiment d'affiliation symbolique.

Le sentiment d'appartenance à la lignée paternelle - tout comme son corolaire, la non-inscription dans la lignée beau-parentale - manifesté par Carla est observé chez tous les enquêtés, indépendamment du jugement qu'ils portent sur la qualité des relations avec leur père, ou bien de la fréquence de leurs contacts, ou encore de la durée de leur vie commune. Ainsi Marcos a un an lorsque ses parents se séparent. Il va alors habiter chez ses grands-parents maternels en compagnie de sa mère jusqu'à ce que, cinq ans plus tard, sa mère vive avec un nouveau compagnon, Mario. C'est à travers la métaphore du don que Marcos exclut son beau-père de son groupe de parenté et affirme son affiliation à son père avec lequel il a peu vécu et qu'il ne rencontre que très rarement :

"Il [Mario] se sentait les droits d'un père et je lui avais même pas donné l'occasion. Il a acheté une bicyclette mais comme si ça allait me tromper, parce que la pression qu'il voulait exercer sur moi et la façon dont il le faisait, tu comprends... j'avais la rage parce que je ne lui donnais pas la liberté d'agir comme ça, je ne lui avais pas donné ce pouvoir. [...] Mon père m’a donné une voiture miniature, il a essayé quelque chose, il n'a pas essayé pour lui, il a acheté un cadeau pour moi, mais mon beau-père ne voulait pas me donner la bicyclette, il voulait m'acheter alors que mon père voulait me faire plaisir, je crois que ça c'est le point essentiel".

Par son cadeau dont il est accusé d'attendre explicitement un retour, Mario établit avec Marcos un rapport de domination personnelle (Bourdieu 1980, 1994). La contrepartie exigée étant jugée excessive, le don ne peut pas être rendu; il contraint Marcos à occuper une position d'éternel débiteur de son beau-père. En acceptant le don, Marcos a contracté une dette morale qu'il ne pourra solder qu'en rompant la relation avec son beau-père; d'ailleurs il se libère de l'obligation de restitution en s'enfuyant de la maison pour se réfugier au domicile de ses grands-parents maternels où il résidera dorénavant. En fait, Mario a transgressé les normes qui régissent la circulation du don dans la 
parenté (Charbonneau 1996) ; c'est ce que démontre la comparaison faite par Marcos entre le cadeau de son beau-père et celui de son père qu'il oppose terme à terme. La petite voiture est certes de moindre valeur marchande que la bicyclette, mais la valeur de la chose n'importe pas au regard de l'esprit dans lequel elle est donnée (Godbout 1992). Alors que le don du père est généreux, au service du lien, motivé par le bonheur du destinataire, celui du beau-père obéit à l'esprit de calcul : intéressé, “inspiré par l'intention d'obliger" (Bourdieu 1980: 180), il vise à créer une dette et exige un dû. Lidéologie du désintéressement des relations familiales est ici au service de l'exclusion du beau-père de la parenté. Marcos refuse explicitement toute transmission venant de son beau-père: "il a essayé de prendre une attitude de père, a priori je l'ai laissé mais il a dépassé les limites parce qu'il voulait que je lui ressemble". Et il conclut en réaffirmant son inscription symbolique dans ses lignées parentales: "certaines choses dans notre vie sont irremplaçables, rien ne peut remplacer un père ou une mère".

L'affiliation symbolique à la lignée paternelle se lit aussi à travers la perception que nos enquêtés ont de leur propre ressemblance familiale. En effet, Bernard Vernier (1999) a brillamment montré que les jugements de ressemblances - physiques et psychologiques - constituent des modalités possibles d'affiliation. Benjamine d'une fratrie de trois enfants, Taís souligne tout au long de son entretien, ses relations affectives privilégiées avec sa mère, Telma, dont elle est la confidente, et sa distance avec son père qu'elle ne voit que très peu depuis que sa mère et ses trois enfants sont allés habiter à Aracaju, peu de temps après la séparation conjugale: "J'ai toujours été très attachée à ma mère, à mon père pas autant, je n'étais pas proche de lui, quand il s'est séparé je me suis séparée bien plus encore. Il me connaît, il me voit comme ça, plus quand je vais là-bas dans l'intérieur, c'est seulement une question de minute, on se voit vite fait". Mais sous le rapport des ressemblances entre elle et les membres de sa famille, elle s'identifie avant tout à son père: "Je pense que je ressemble plus à mon père qu'à ma mère. Je suis très coléreuse et lui aussi. Mon frère l'est un peu mais ma sœur pas du tout... Il n'est pas très affectueux, seulement quand il a bu, moi non plus je ne suis pas très affectueuse". En fait, Taís compense doublement sa distance affective vis-à-vis de son père par une proximité symbolique: d'une part en mentionnant qu'elle ressemble davantage à son père qu'à sa mère; d'autre part en déclarant que c'est elle qui, au sein de sa fratrie, a le plus hérité des caractéristiques psychologiques de son père. Tout se passe comme si la proximité symbolique était inversement proportionnelle à la proximité affective: son frère et sa sœur qui ont davantage vécu avec leur père, tant avant (ils sont les ainés de Taís) qu'après la dissolution de l'union (ne s'habituant pas à la vie à Aracaju, ils sont retournés vivre au bout d'un an et demi dans l'intérieur, résidant alternativement chez leur père et leur grandmère maternelle), peuvent finalement ne pas ressembler à leur père sans pour 
autant que leur filiation soit menacée ; par contre pour Taís, sa ressemblance à son père a une fonction pratique : elle la relie à sa lignée paternelle. Elle affirme d'ailleurs: "malgré tout ce qu'il a fait... se disputer avec ma mère, mais il est mon père".

Nous n'avons donc pas observé dans notre enquête, une quelconque " désaffiliation” des enfants envers leur lignée paternelle. Ce constat s'écarte des résultats de travaux réalisés en France (Théry 1998; Cadolle 2000 ; Martial 2003). Il peut s'agir d'une différence de points de vue: nous ne nous intéressons ici qu'aux sentiments de parenté et, en l'occurrence, aux sentiments d'affiliation symbolique des beaux-enfants, tandis que les recherches françaises ont tendance à prendre comme indicateur de désaffiliation (mais aussi de maintien du lien de filiation), l'absence (mais aussi la fréquence) de contacts entre le père et ses enfants, et/ou le (non-)versement d'une pension alimentaire, et à déduire ainsi la filiation de la parentalité. Or, il convient de différencier très nettement, au risque de confondre des réalités de nature différente, ce qui relève de l'appartenance à un groupe et de l'identité sociale des enfants (la filiation) et ce qui relève de l'exercice des fonctions parentales (la parentalité).

On ne peut, certes, manquer de souligner qu'après la séparation du couple, les contacts avec le père sont globalement sporadiques, mais les liens ne sont néanmoins pas totalement rompus; de même, et de façon plus générale, les échanges avec la lignée paternelle sont beaucoup moins fréquents et moins intenses affectivement qu'avec la lignée maternelle, comme ils l'étaient déjà d'ailleurs pendant la vie commune. On observe aussi après la dissolution de l'union, un net désengagement financier des pères vis-à-vis de leurs enfants avec lesquels ils ne résident plus. Durant la période de cohabitation, les dépenses des enfants étaient assumées par le père, si sa conjointe ne travaillait pas, ou bien, dans le cas contraire, partagées entre les deux parents. Après la séparation, seulement un tiers des pères de notre échantillon participe à l'entretien de leurs enfants, ainsi que nous l'indiquions précédemment. Notons que le versement d'une pension alimentaire est le plus souvent obtenu par coercition, et non payé volontairement.

Toutefois, il importe de ne pas surestimer les changements qu'occasionne la rupture conjugale sur le lien père-enfant et les sentiments d'affiliation symbolique. La dissolution de l'union ne signifie pas systématiquement une profonde redéfinition des relations entre les enfants et leur père. Ainsi, la séparation consensuelle des parents de Carla que nous évoquions plus haut passe inaperçue à ses yeux de fillette de quatre ans, son père lui rendant visite très fréquemment: "il était toujours à la maison, il venait... alors je n'ai pas pris ça pour une séparation”. Par contre, le départ de ce dernier quelques mois plus tard, vers une autre région du pays située à plusieurs milliers de kilomètres l'afflige profondément (“ça, ça a été un drame pour moi quand il a déménagé parce que je ne le voyais plus"); c'est à cette occasion seulement que se produit leur 
séparation. Si certains pères restent très présents dans la vie de leur progéniture même après la rupture de l'union, d'autres, au contraire, sont absents du quotidien des enfants bien avant la séparation conjugale. D'une part, du fait d'une rigide division du travail au sein des couples y compris biactifs, qui attribue soins et éducation de la progéniture à la mère et la fonction de pourvoyeur des revenus familiaux au père. Cette situation a très bien été décrite par Blöss (1996) sous le terme de "monoparentalité éducative": même pendant la vie commune avec ses enfants, le père exerce "de fait une fonction paternelle symbolique plus que pratique” (Blöss 1996: 53). Ainsi certains de nos interlocuteurs ne voyaient leur père souvent occupé à l'extérieur du foyer, que le dimanche. Le témoignage de Sandro est à ce titre éloquent :

"- Comment étaient tes relations avec ton père avant la séparation?

- Comme après la séparation, mon père a toujours beaucoup travaillé, alors il avait du temps ni pour moi ni pour ma mère, il a toujours été un père qui travaillait beaucoup, il a toujours donné la priorité à son travail, nous nous voyions très peu mais pendant le temps que nous passions ensemble il aimait me donner cette attention... me dire 'regarde je suis un père présent', il essayait de courir derrière le temps perdu mais il n'y arrivait pas. C'était une bonne relation mais bien petite, bien peu".

D'autre part, un quart de nos interlocuteurs n'a jamais cohabité avec leur père, leurs parents se séparant avant leur naissance. ${ }^{17}$ Certains d'entre eux n'ont d'ailleurs connu leur père que tardivement, comme Fabiana qui vit depuis l'âge de 4 ans avec son beau-père, Francisco. Ses parents se sont séparés pendant la grossesse de sa mère. Son père Fabio, peu convaincu de sa paternité, ne la reconnaît que sous la contrainte: "il a été obligé de me reconnaitre, parce que mon grand-père [maternel] lui a mis une arme sur la tête... Mais il n'a jamais rien cherché à savoir et ma mère ne lui a jamais demandé d'argent ou quoi que ce soit non plus, elle ne l'a jamais mis en justice..." Fabiana a 12 ans lorsque Fabio lui rend visite pour la première fois, en compagnie de son épouse et de son jeune fils qui vient de naître. En prenant l'initiative d'une rencontre avec Fabiana et en la conviant chez lui - invitation qu'elle accepte en se rendant quelques semaines plus tard chez son père -, Fabio démontre qu'il l'accepte comme fille, qu'il la reconnaît comme sienne; c'est par un acte volontaire, intentionnel, opposé à la reconnaissance juridique contrainte, que s'établit pleinement la filiation de Fabiana. Depuis lors, leurs contacts sont irréguliers mais non inexistants; lorsque Fabiana, jeune étudiante aux revenus modestes, a besoin d'argent, elle sollicite en premier lieu sa mère, puis son père,

17 Au total, un peu plus de la moitié de nos enquêtés a cessé de vivre avec leur père avant l'âge de deux ans. 
mais jamais son beau-père. L'inscription symbolique de Fabiana se manifeste à l'égard de son père et aucunement à l'égard de son beau-père qui l'a pourtant élevé pendant plus de 14 ans (“je crois qu'un beau-père ne va jamais réussir à aimer une personne qui n'est pas son enfant, autant qu'il aime un enfant”).

Enfin, même en l'absence de contacts directs entre le père et ses enfants, différents mécanismes prescrivent à l'enfant sa filiation paternelle, y compris après la dissolution de l'union. Ainsi les échanges avec des membres de la famille paternelle sont parfois maintenus, même s'ils sont moins fréquents ou jugés moins intenses affectivement qu'avec la lignée maternelle. A cet égard, les femmes (mères, grand-mère ou encore tante paternelles) jouent un rôle central pour assurer la continuité des relations familiales après la rupture conjugale; différentes recherches réalisées auprès de couples non-séparés les désignent d'ailleurs comme les principales gestionnaires des contacts non seulement avec leur propre famille, mais aussi avec celle de leur conjoint (voir entre autres Coenen-Huther, Kellerhals et Von Allmen 1994; Schwartz 2002 [1990]; Attias-Donfut, Lapierre et Segalen 2002 ; Kellerhals et Languin 2008 ; Clément 2009). C'est à l'initiative de sa tante paternelle et avec l'accord de sa mère que Suzana connaît, à l'âge de 8 ans, son père ainsi que les parents de ce dernier; Suzana n'aura par la suite que des contacts très épisodiques avec son père mais maintient ce lien tenu avec sa lignée paternelle en rendant régulièrement visite à sa tante. Enfin, comme le notait Françoise Héritier-Augé à propos des Samo du Burkina-Faso, "la parole crée la filiation au même titre que le sang” (1985: 14); c'est aussi à travers les récits et souvenirs des membres de la famille paternelle et maternelle - et notamment de la mère, parfois nostalgique de ses premiers amours de jeune-fille -, que le père continue à être présent symboliquement auprès de l'enfant.

\section{CONCLUSION: À PROPOS DE LA PARENTÉ VOLONTAIRE}

Au terme de ce cheminement qui nous a conduits à parcourir les logiques argumentatives utilisées par les beaux-enfants pour évoquer la teneur de leur relation avec leur beau-père, nous pouvons retenir que l'affiliation volontaire des beaux-enfants a pour support le partage du quotidien et l'affinité ; l'affiliation symbolique quant à elle, est toujours réservée à la lignée paternelle, même si les relations avec le père sont distendues et jugées peu satisfaisantes. Toutefois, la reconnaissance par les beaux-enfants de liens filiaux avec le conjoint de leur mère repose sur une double condition: d'une part que le beau-père soit investi par la mère et le père (si ce dernier est présent dans la vie de l'enfant) de devoirs et de droits parentaux, d'autre part qu'il sanctionne cette investiture en s'appropriant ces devoirs et droits. Cependant cette double condition est très rarement réalisée dans les familles que nous avons enquêtées. En effet, on ne peut manquer d'observer à partir des entretiens menés auprès des adultes 
que les beaux-pères manifestent une tendance à la réserve vis-à-vis de leurs beaux-enfants, voire une résistance à assumer un rôle parental. Mais surtout, les mères se montrent peu disposées à confier leurs droits et responsabilités à leur conjoint qu'elles maintiennent directement (dans les classes moyennes) ou indirectement (dans les classes populaires) à distance des décisions relatives à leurs enfants. Elles justifient leur mise à l'écart de leur conjoint par l'absence de lien biologique: seul le partage du sang crée un réel attachement affectif capable de tempérer le caractère nécessairement coercitif de l'éducation. Le décès du père est l'unique situation susceptible de rendre légitime l'exercice d'une autorité parentale par le beau-père ; une substitution pourrait alors être envisagée, mais à la condition que l'enfant soit élevé dès son plus jeune âge par le beau-père ; la formule additive, on l'aura remarqué, n'est pas retenue. On peut alors s'étonner d'entendre les mères affirmer de façon réitérée que leur conjoint est comme un père pour leurs enfants. Le fait que ce dernier subvienne indirectement aux besoins de ses beaux-enfants en participant aux dépenses communes de la maisonnée ${ }^{18}$ alors qu'il est dégagé de toute obligation d'entretien à leur égard, mais aussi l'absence de manifestations d'animosité du premier envers les seconds sont les deux éléments sur lesquels les mères se basent pour percevoir leur partenaire "comme un père". A contrario, les seules situations où les beaux-enfants se reconnaissent un lien familial avec leur beau-père se produisent lorsque la mère a explicitement délégué ses prérogatives parentales au beau-père, et que celui-ci accepte de façon expresse de prendre en charge matériellement et affectivement l'enfant de sa conjointe qu'il "adopte" selon l'expression employée par une des belles-filles interrogées ; tout se passe comme si les enfants ne pouvaient développer des sentiments filiaux à l'égard de leur beau-père que sur la base des volontés conjointes de ses parents et de son beaupère. Ces éléments nous permettent maintenant de comprendre pourquoi des formes d'affiliation volontaire se développent dans le cadre du fosterage ou de l'adoption (légale ou non), à l'égard des parents sociaux, alors que, dans le cadre de la recomposition familiale, les enfants n'éprouvent que très rarement des sentiments filiaux pour leur beau-père. En fait, le transfert d'enfants passe par la volonté des donneurs de se dessaisir de leurs droits parentaux au profit d'autrui, et celle des récipiendaires ${ }^{19}$ de prendre en charge les enfants d'un tiers. Or cette conjonction des volontés est très peu fréquente dans nos entretiens, ce qui conduit les beaux-enfants à rejeter leur beau-père hors de leur parenté.

18 Les entretiens réalisés avec les beaux-pères et les mères montrent que les dépenses alimentaires et d'habitation sont partagées entre les conjoints, selon des modes de calcul variés, égalitaires ou non, qui peuvent être basés sur les ressources ou bien sur les charges de chacun, mais que les intéressés jugent dans l'ensemble équitables. Les mères participent donc toujours aux dépenses communes, contrairement à la norme morale énoncée par leur progéniture.

19 Sans préjuger, bien sûr, des contraintes qui peuvent peser sur les uns ou sur les autres dans l'échange. 


\section{BIBLIOGRAPHIE}

ABREU, Domingos, 2002, No Bico da Cegonha: Histórias de Adoção e da Adoção Internacional no Brasil. Rio de Janeiro, Relume-Dumará.

ANDERLE, Elisabeth Nass, 2002, "A posse de estado de filho e a busca pelo equilíbrio das verdades da filiação", disponible à <http://jus2.uol.com.br/doutrina/texto.asp?id $=3520>$ (accès 05-08-2009).

ATTIAS-DONFUT, Claudine, Nicole LAPIERRE, et Martine SEGALEN, 2002, Le nouvel esprit de famille. Paris, Ed. Odile Jacob.

BLÖSS, Thierry, 1996, Éducation familiale et beau-parenté : l'empreinte des trajectoires biographiques.

Paris, L'Harmattan, coll. Logiques Sociales.

BOURDIEU, Pierre, 1980, Le sens pratique. Paris, Éditions de Minuit.

—, 1994, Raisons pratiques : Sur la théorie de l'action. Paris, Seuil.

BOZON, Michel, 1990, “Les femmes et l'écart d'âge entre conjoints: une domination consentie. II. Modes d'entrée dans la vie adulte et représentations du conjoint”, Population, 3: 565-601.

CADOLLE, Sylvie, 2000, Etre parent, être beau-parent: La recomposition de la famille. Paris, Ed. Odile Jacob.

— , 2005, “'C'est quand même mon père!' La solidarité entre père divorcé, famille paternelle et enfants adultes", Terrain, 45: 83-96.

CHARBONNEAU, Johanne, 1996, "Le côté sombre du don dans la parenté", Recherches Sociographiques, 37 (1): 113-130.

CLÉMENT, Céline, 2009, La mère et ses enfants : Devenir adulte et transmissions intergénérationnelles. Paris, L'Harmattan.

COENEN-HUTHER, Josette, Jean KELLERHALS, et Malik VON ALLMEN, 1994, Les réseaux de solidarité dans la famille. Lausanne, Éditions Réalités Sociales.

FINE, Agnès (dir.), 1998, Adoptions: Ethnologie des parentés choisies. Paris, Éditions de la Maison des Sciences de l'Homme.

— 2001 , "Vers une reconnaissance de la pluriparentalité?", Esprit, 273 : 40-52.

FONSECA, Claudia, 2002, "Mãe é uma só? Reflexões em torno de alguns casos brasileiros", disponible à <www.scielo.br/scielo.php?script=sci_arttext\&pid=S0103-65642002000 $200005 \& \operatorname{lng}=$ pt\&nrm $=$ iso $>$ (accès 20-04-2008).

— - 2006a, "Da circulação de crianças à adoção internacional: questões de pertencimento e posse", Cadernos Pagu, 26: 11-43.

—, 2006b [1995], Os Caminhos da Adoção. São Paulo, Cortez.

GODBOUT, Jacques, 1992, L'esprit du don. Paris, La Découverte et Montréal, Boréal, avec la collaboration d'Alain Caillé.

HÉRITIER-AUGÉ, Françoise, 1985, “La cuisse de Jupiter”, L’Homme, 25 (94): 5-22.

JOHNSON, Colleen L., 2000, “La réorganisation de la parenté aux Etats-Unis après le divorce et le remariage”, Anthropologie et Sociétés, 24 (3): 93-1 14.

KELlerhalls, Jean, et Noëlle LANGUIN, 2008, Juste? Injuste? Sentiments et critères de justice dans la vie quotidienne. Paris, Payot.

LALlEMAND, Suzanne, 1993, La circulation des enfants en société traditionnelle: Prêt, don, échange. Paris, L'Harmattan.

LE GALL, Didier, et Yamina BETTAHAR, 2001, La pluriparentalité. Paris, PUF.

LÔBO, Paulo Luiz Netto, 2003, “Direito ao estado de filiação e direito à origem genética: uma distinção necessária”, disponible à <http://jus2.uol.com.br/doutrina/texto.asp?id= $4752 \&>$ (accès 10-08-2009). 
MARTIAL, Agnès, 2003, S'apparenter. Paris, Éditions de la Maison des Sciences de l'Homme.

— 2005 , "Comment rester liés? Les comptes des familles recomposées", Terrain, 45: 67-82. MORENO, Alessandra Zorzetto, 2007, "Vivendo em Lares Alheios": Acolhimento Domiciliar, Criação e Adoção na Cidade de São Paulo (1765-1822). Campinas, Unicamp, thèse de doctorat.

SCHWARTZ, Olivier, 2002 [1990], Le monde privé des ouvriers. Paris, PUF.

SEGALEN, Martine, 2000, "Enquêter sur la grand-parentalité en France”, Anthropologie et Sociétés, 24 (3): 75-91.

SERRA, Márcia Milena Pivatto, 2003, O Brasil de Muitas Mães: Aspectos Demográficos da Circulação de Crianças. São Paulo, Universidade Estadual de Campinas, thèse de doctorat.

SINGLY, François de, 1994 [1987], Fortune et infortune de la femme mariée: Sociologie des effets de la vie conjugale. Paris, PUF.

THÉRY, Irène, 1998, Couple, filiation et parenté aujourd'hui: Le droit face aux mutations de la famille et de la vie privée. Paris, Odile Jacob.

VERNIER, Bernard, 1999, Le visage et le nom: Contribution à l'étude des systèmes de parenté. Paris, PUF.

WEBER, Florence, 2002, “Pour penser la parenté contemporaine: maisonnée et parentèle, des outils de l'anthropologie", en Danièle Debordeaux et Pierre Strobel, Les solidarités familiales en questions: Entraide et transmission. Paris, L. G. D. J., 73-106.

— - 2003, "Modéliser l'économie domestique”, en Florence Weber, Séverine Gojard et Agnès Gramain (dirs.), Charges de famille: Dépendance et parenté dans la France contemporaine. Paris, La Découverte, 9-42.

— 2005 , Le sang, le nom et le quotidien: Une sociologie de la parenté pratique. Paris, Aux Lieux d'Être.

— tidienne", Informations Sociales, 3: 30-41.

—, 2006b, "Lares de cuidado e linhas de sucessão: algumas indicações etnográficas na França, hoje”, Mana, 12 (2): 479-502.

YNGVESSON, Barbara, 2007, "Parentesco reconfigurado no espaço da adoção", Cadernos Pagu, 29: $111-138$.

Quando o marido da minha mãe não é meu pai: enteados e padrastos no Brasil contemporâneo - Christine Jacquet - Universidade Federal de Sergipe, Brasil • jojokiki@ig.com.br.

Baseado na abordagem desenvolvida por Florence Weber sobre o parentesco prático, esse artigo tenta identificar a natureza do vínculo entre padrastos e enteados no Brasil, apresentando o sistema de justificação adotado por esses últimos para evocar os sentimentos que nutrem para com seu padrasto. A singularidade do parentesco brasileiro - que não assenta a definição do parente apenas sobre a consanguinidade e admite o exercício efetivo do pluriparentesco - incentivava a pensar a priori que a partilha do cotidiano era susceptível de gerar entre os enteados sentimentos familiares; no entanto, os resultados da pesquisa infirmaram essa hipótese inicial.

PALAVRAS-CHAVE: recomposição familiar, parentesco prático, sentimentos de parentesco; afiliação voluntária. 